\title{
Offset Ruled Surface in Euclidean Space with Density
}

\author{
Neslihan Ulucan and Mahmut Akyigit
}

\begin{abstract}
In this paper, offset ruled surfaces in these spaces are defined by using the geometry of ruled surfaces in Euclidean space with density. The mean curvature and Gaussian curvature of these surfaces are studied. In addition, the relationships between the mean curvature and mean curvature with density, and the Gaussian curvature and the Gaussian curvature with density of the offset ruled surfaces in $E^{3}$ with density $e^{z}$ and $e^{-x^{2}-y^{2}}$ are given.
\end{abstract}

\section{Introduction}

Surfaces have important applications in many disciplines such as computer graphics, engineering, and physics. The subject of ruled surfaces, which has a lot of usage and application area among the surface subjects, was first studied by G. Monge. These surfaces are the result of the continuous movement of a line along the space curve called the reference curve in 3-dimensional Euclidean space. Classification of ruled surfaces, properties of reference curve, geodesics, shape operator of the surface, developable surfaces, investigation of non-developable ruled surfaces are among the leading studies on ruled surfaces.

The topic of manifolds with density, which has been studied in geometry in recent years, has become widely available in mathematics. If we consider physical areas and surfaces with different density, we will come across this subject.

Key Words: Ruled surface, Offset surface, Space with density.

2010 Mathematics Subject Classification: 53A05; 14J26; 14 Q10.

Received: 01.04.2020

Accepted: 25.05.2020 
Density in a Riemannian manifold is defined as a positive function that weights both volume and surface area. F. Morgan, who has many studies on the subject, defined the mean and Gaussian curvature of manifolds with density in studies [1]-[3], examined Perelman's proof of Poincaré hypothesis on surfaces with density and generalized Myers theorem to Riemannian manifolds with density. Many studies have been made surfaces with density based on the definitions of F. Morgan. C. Rosales studied the isoperimetric problem of Euclidean space with continuous density and characterized the isoperimetric regions for uniform density in one dimension, [4]. I. Corwin described the Gauss-Bonnet formula of a manifold with density, geodesics and constant surface curvature, [5]. Density function has also been highly studied on minimal surfaces. L. Belarbi expressed the equations of linearly minimal surfaces with density in Euclidean space and characterized the solutions of equations of some minimal graphs. It also examined density Gaussian and mean curvatures for surfaces with radial density, [6]. R. Lopez classified all minimal surfaces in Euclidean space with logarithmic linear density, [7]. D. S. Kim classified the mean and Gaussian curvatures by exemplifying weight minimal helicoidal surfaces on surfaces with density for helicoidal surfaces, [8]. D. T. Hieu showed the existence of a family of cylindrical and non-cylindrical ruled minimal surfaces with density by classifying density ruled minimal surfaces, [9]. For more details on surfaces in manifolds with density, see [10]-[14].

One of the most important examples of a 2-dimensional surface with density is the Gauss plane. This plane is an Euclidean plane with length and volume weighted by $(2 \pi)^{-1} e^{-r^{2} / 2}$, where $r$ is the distance from the origin. By using perimeter and weight volume, smooth Riemannian manifolds with a smooth positive density $e^{\phi(x)}$ can be considered.

Generally, one can define the new weighted area and volume as

$$
\begin{aligned}
& d P \phi=e^{\phi} \cdot d P \\
& d V \phi=e^{\phi} \cdot d V
\end{aligned}
$$

where $d P$ is underlying Riemannian perimeter and $d V$ is underlying Riemannian volume for a manifold with density, [5]. From now on, new functions which are Gaussian and mean curvatures of a Riemannian surface with density by utilizing the density function can be defined.

Definition 1. Gaussian curvature $K_{\phi}$ of a Riemannian surface with density $e^{\phi}$ is given by

$$
K_{\phi}=K-\Delta \phi
$$

where $K$ is the Riemannian Gaussian curvature and $\Delta$ is the Laplacian operator, [5]. 
Definition 2. Mean curvature $H_{\phi}$ of the Euclidean 3-space with density $e^{\phi}$ is given by

$$
H_{\phi}=H-\frac{1}{2}\langle\boldsymbol{N}, \nabla \phi\rangle
$$

where $H$ and $\boldsymbol{N}$ are the mean curvature and the unit normal vector of the surface, and $\nabla \phi$ is the gradient of $\phi,[5]$

We denote the Gaussian curvature with density or Gaussian $\phi$-curvature of the surface with $K_{\phi}$, and the mean curvature with density or mean $\phi$-curvature of the surface with $H_{\phi}$. These curvatures will be shown as the Gaussian $\phi$ curvature and the mean $\phi$-curvature throughout the article.

\section{Preliminaries}

A ruled surface $M$ in Euclidean 3 -space $E^{3}$ is a surface that can be swept out by a straight line parallel to $\beta$ along a curve $\alpha$. It has a parametrization of the form

$$
X(u, v)=\alpha(u)+v \beta(u), \quad u \in I_{1} \subset R, \quad v \in I_{2} \subset R,
$$

where the curve $\alpha=\alpha(u)$ is called base curve (also called ruled surface directrix) and $\beta=\beta(u)$ is the director curve of the ruled surface. The rulings of a ruled surface are asymptotic curves.

We characterize some special types of ruled surface, choosing $\alpha$ and $\beta$. For example, if $\beta$ is constant, the ruled surface is said to be cylindrical, and non-cylindrical otherwise. It is well known that cylindrical ruled surfaces are developable. In that case, the Gaussian curvature of the ruled surface is zero. We will focus on non-developable ruled surfaces.

Let $M$ be a non-developable ruled surface in $E^{3}$. In this case, the parametrization for surface $M$ is given by

$$
\varphi(u, v)=c(u)+v e(u)
$$

such that $\langle e(u), e(u)\rangle=\left\langle e^{\prime}(u), e^{\prime}(u)\right\rangle=1$, and $\left\langle c^{\prime}(u), e^{\prime}(u)\right\rangle=0$.

Under the circumstances the parameter $u$ is the arc-length on spherical curve $e(u)$ and $c(u)$ is the striction curve of the ruled surface $\phi$. We can assume that $e$ curve is a vector and this curve called a spherical indicator vector of surface $\varphi$.

Let $t=e^{\prime}$ and $g=e \times e^{\prime}$. The orthonormal frame along with the curve $e$ in $E^{3}$ is $\{e, t, g\}$ where is called the spherical Frenet frame of spherical curve $e$. The central and asymptotic normals of $\varphi(u, v)$ are said to be $t$ and $g$, respectively, [15]. 
For the spherical Frenet frame $\{e, t, g\}$, the following equations hold:

$$
\begin{aligned}
e^{\prime} & =t \\
t^{\prime} & =-e-J g \\
g^{\prime} & =J t
\end{aligned}
$$

where $J=\left\langle e^{\prime \prime}, e^{\prime} \times e\right\rangle$ denotes the geodesic curvature of $\kappa_{g}$ of a spherical indicatrix curve $e,[15]$. Also, the derivative of the striction curve $c$ is given by

$$
c^{\prime}=F e+Q g .
$$

Then, with the help of the equations (5) and (6), the components of the first fundamental form of the ruled surface are given by

$$
E=v^{2}+F^{2}+Q^{2}, \quad F=\left\langle c^{\prime}, e\right\rangle, \quad G=1 .
$$

The structure functions $F$ and $Q$ of $\varphi$ satisfy $F^{2}+Q^{2}=1$ if the parameter $u$ is also the arc-length parameter of the striction curve $c$ of $\varphi$.

Moreover the unit normal vector $\boldsymbol{u}$ of $\varphi$ is written as

$$
\boldsymbol{u}=\frac{Q t-v g}{D}
$$

where $D=\sqrt{E G-F^{2}}=\sqrt{v^{2}+Q^{2}}$.

The components of the second fundamental form of the ruled surface $\varphi$ are given by $L=\frac{Q(F+Q J)-Q^{\prime} v+J v^{2}}{D}, \quad M=\frac{Q}{D} \neq 0, \quad N=0$. With the help of the coefficients of the fundamental forms, Gaussian and mean curvatures of ruled surface $\varphi$ are

$$
\begin{aligned}
& K=-\frac{Q^{2}}{D^{4},} \\
& H=\frac{Q(Q J-F)-Q^{\prime} v+J v^{2}}{2 D^{3}},
\end{aligned}
$$

respectively. Due to the fact that $\varphi$ is a non-developable ruled surface, the function $Q$ is non zero everywhere, [15]. The detailed information related to offset surfaces can be found in [15]-[16].

Definition 3. Let $\varphi$ and $\varphi^{*}$ be two ruled surfaces in $E^{3}$. The surface $\varphi$ is said to be an involute offset of $\varphi^{*}$ if there exists a one-to-one correspondence between their rulings such that the central normal of $\varphi$ and the spherical indicatrix vector of $\varphi^{*}$ are linearly dependent at the striction points of their corresponding rulings, [15]. 
Then the surface $\varphi^{*}$ can be written as the equation (4)

$$
\varphi^{*}(u, v)=c^{*}(u)+v e^{*}(u)=c(u)+R(u) t(u)+v(u) t(u) .
$$

Here, $c^{*}(u)$ is the striction curve and $e^{*}=e^{*}(u)$ is the director curve of the offset ruled surface $\varphi^{*}$. Also, $R$ is the distance between the corresponding striction points of $\varphi$ and $\varphi^{*}$.

By using (5) and (6), the coefficients of the first fundamental form of $\varphi^{*}$ are

$$
\begin{aligned}
& E^{*}=Q^{2}+F^{2}-2(R+v)(F+J Q)+(R+v)^{2}\left(1+J^{2}\right)+R^{\prime 2} \\
& F^{*}=R^{\prime} \\
& G^{*}=1 .
\end{aligned}
$$

The coefficients of the second fundamental form as follows:

$$
\begin{aligned}
& L^{*}=\frac{(-Q+R J+J V)\left(F-2 R^{\prime}\right)+(F-R-v)\left(Q-2 R J-J^{\prime}(R+V)\right)}{D^{*}} \\
& M^{*}=\frac{Q-F J}{D^{*}} \\
& N^{*}=0
\end{aligned}
$$

where $D^{*}=\sqrt{(-Q+R J+v J)^{2}+(F-R-v)^{2}}$. Also, the unit normal vector of $\boldsymbol{u}^{*}$ of $\varphi^{*}$ is given by

$$
\boldsymbol{u}^{*}=\frac{1}{D^{*}}[(-Q+R J+v J) e+(F-R-v) g] .
$$

By the help of the coefficients of the fundamental forms, Gaussian and mean curvatures of offset ruled surface $\varphi^{*}$ are respectively,

$$
\begin{aligned}
& K^{*}=-\frac{(Q-F J)^{2}}{D^{* 4}} \\
& H^{*}=\frac{H_{1}{ }^{* 3}}{2 D^{* 3}}
\end{aligned}
$$

where

$H_{1}{ }^{*}=\left(R^{2} J^{\prime}+F Q^{\prime}+F^{\prime} J R-F R J^{\prime}-R Q^{\prime}-F^{\prime} Q\right)+\left(2 R J^{\prime}+F^{\prime} J-F J^{\prime}-Q^{\prime}\right) v+J^{\prime} v^{2}$, $[15]$. 


\section{Offset Ruled Surface in Euclidean Space $E^{3}$ with Den- sity}

In this section, by using the definition of ruled surface in space with density, we define involute offset surface of ruled surface in space with density. Moreover, we study Gaussian and mean curvatures of ruled surface and offset ruled surface in space with densities $e^{z}$ and $e^{-x^{2}-y^{2}}$. In addition, the relations between the Gaussian curvature and Gaussian curvature with density, and mean curvature and mean curvature with density of offset ruled surfaces in space with density are given.

Definition 4. Let $\varphi$ and $\varphi^{*}$ be two ruled surfaces in space with density. The surface $\varphi$ is said to be an involute offset of $\varphi^{*}$ in space with density if there exists a one-to-one correspondence between their rulings such that the central normal of $\varphi$ and the spherical indicatrix vector of $\varphi^{*}$ are linearly dependent at the striction points of their corresponding rulings.

Then, with the help of this definition, we can express Gaussian and mean curves of offset ruled surfaces in spaces with different densities.

\subsection{Offset ruled surface in space with density $e^{z}$}

In this section, we can assume that $e^{z}$ is density of Euclidean space containing the ruled surfaces $\varphi(u, v)$ and $\varphi^{*}(u, v)$.

We show the relationships between the Gaussian curvature and the Gaussian $\phi$-curvature, and the mean curvature and mean $\phi$-curvature of the offset ruled surfaces in space with density $e^{z}$.

Gaussian curvature of offset ruled surface $\varphi^{*}(u, v)$ in space with density $e^{z}$ is

$$
K^{*}=-\frac{1}{D^{* 4}}(Q-F J)^{2}
$$

where $D^{*}=\sqrt{(-Q+R J+v J)^{2}+(F-R-v)^{2}}$.

By using the equation (1), Gaussian $\phi$-curvature of offset ruled surface $\varphi^{*}(u, v)$ in space with density $e^{z}$ in terms of structure functions $J, F$ and $Q$ is found

$$
K_{\phi}^{*}=-\frac{1}{D^{* 4}}(Q-F J)^{2}-\Delta_{\phi}
$$

Since $\phi=z$ is a density function, $\nabla \phi=(0,0,1)$. Therefore, $\Delta_{\phi}=0$. From (11) and (12), we obtain 


$$
K_{\phi}^{*}=K^{*} .
$$

Then about calculation, we give the following theorem.

Theorem 1. Let $\varphi^{*}$ be an offset ruled surface of ruled surface $\varphi$ in Euclidean 3-space with density $e^{z}$. Also, $K^{*}$ and $K_{\phi}^{*}$ be Gaussian curvature and Gaussian $\phi$-curvature of offset ruled surface in space with density, respectively. Then, the Gaussian $\phi$-curvature $K_{\phi}^{*}$ of offset ruled surface $\varphi^{*}$ is equal to Gaussian curvature $K^{*}$ of the same surface.

The following remark can be given with the help of equations (11) and (12).

Remark 1. The Gaussian curvature and the Gaussian $\phi$-curvature of offset ruled surface $\varphi^{*}(u, v)$ in space with density are the same as a constant difference.

Theorem 2. Let $\varphi^{*}$ be an evolute offset of a non developable ruled surface $\varphi$. If $Q, J$ and $F$ structure functions of surface $\varphi$ provide $Q=J F$ condition, $\varphi^{*}$ has a zero Gaussian curvature in space with density.

We will show mean $\phi$-curvature $H_{\phi}$ of non-developable ruled surface $\varphi(u, v)$ and mean $\phi$-curvature $H_{\phi}^{*}$ of offset ruled surface $\varphi^{*}(u, v)$ in space with density $e^{z}$.

Now, by using the equations (2), (7) and (8), the mean $\phi$-curvature $H_{\phi}$ of non-developable ruled surface $\varphi(u, v)$ in space with density $e^{z}$ in terms of structure functions $Q, J$ and $F$ is

$$
\begin{aligned}
H_{\phi} & =\frac{J v^{2}-Q^{\prime} v+Q(Q J-F)}{2 D^{3}}-\frac{1}{D}\langle(0,0,1), Q t-v g\rangle \\
& =\frac{J v^{2}-Q^{\prime} v+Q(Q J-F)-2 D^{2}\left[Q t_{3}-v g_{3}\right]}{2 D^{3}} .
\end{aligned}
$$

By using the equations (2), (9) and (10), the mean $\phi$-curvature $H_{\phi}^{*}$ of offset ruled surface $\varphi^{*}(u, v)$ in space with density $e^{z}$ in terms of structure function $Q, J$ and $F$ is

$$
\left.\begin{array}{rl}
H_{\phi}^{*} & =\frac{1}{2 D^{* 3}}\left[\begin{array}{c}
\left(R^{2} J^{\prime}+F Q^{\prime}+F^{\prime} J R-F R J^{\prime}-R Q^{\prime}-F^{\prime} Q\right) \\
+\left(2 R J^{\prime}+F^{\prime} J-F J^{\prime}-Q^{\prime}\right) v+J^{\prime} v^{2}
\end{array}\right] \\
- & \frac{1}{2}\left\langle(0,0,1), \frac{1}{D^{*}}[(-Q+J R+J v) e+(F-R-v) g]\right\rangle .
\end{array}\right]
$$

Let $g=\left(g_{1}, g_{2}, g_{3}\right)$ be an asymptotic normal of offset ruled surface $\varphi^{*}(u, v)$ in space with density $e^{z}$ and $e=\left(e_{1}, e_{2}, e_{3}\right)$. Thus, we have 


$$
\left.\begin{array}{rl}
H_{\phi}^{*} & =\frac{1}{2 D^{* 3}}\left[\begin{array}{c}
\left(R^{2} J^{\prime}+F Q^{\prime}+F^{\prime} J R-F R J^{\prime}-R Q^{\prime}-F^{\prime} Q\right) \\
+\left(2 R J^{\prime}+F^{\prime} J-F J^{\prime}-Q^{\prime}\right) v+J^{\prime} v^{2}
\end{array}\right] \\
-\frac{1}{2 D^{*}}\left[(-Q+J R+J v)\left(e_{3}\right)+(F-R-v)\left(g_{3}\right)\right] .
\end{array}\right]
$$

\subsection{Offset ruled surface in space with density $e^{-x^{2}-y^{2}}$}

In this section, we will examine Gaussian and mean $\phi$-curvatures of ruled surfaces $\varphi(u, v)$ and $\varphi^{*}(u, v)$ in space with density $e^{-x^{2}-y^{2}}$.

Now, by using the equation (4), we show Gaussian $\phi$-curvature $K_{\phi}^{*}$ of offset ruled surface $\varphi^{*}$. Since $\phi=-x^{2}-y^{2}, \Delta_{\phi}=-4$. From equation (1), Gaussian $\phi$-curvature of offset ruled surface in space with density is

$$
K_{\phi}^{*}=-\frac{1}{D^{* 4}}(Q-F J)^{2}+4
$$

Theorem 3. Let $\varphi^{*}$ be an offset ruled surface of non-developable ruled surface $\varphi$ in Euclidean 3-space $E^{3}$ with density. Also, let $e^{-x^{2}-y^{2}}$ be density of Euclidean space containing the offset ruled surface $\varphi^{*}$. If $\varphi^{*}$ is developable, then

$$
2 D^{* 2}=\mp(Q-F J) \text {. }
$$

Now, we investigate mean $\phi$-curvature $H_{\phi}$ of non-developable ruled surface $\varphi$ and its minimal state. Let $t=\left(t_{1}, t_{2}, t_{3}\right)$ and $g=\left(g_{1}, g_{2}, g_{3}\right)$ be normal vector and asymptotic vector of ruled surface $\varphi$ in space with density. Due to density $e^{-x^{2}-y^{2}}$ of Euclidean space containing the offset ruled surface $\varphi$, $\nabla_{\phi}=(-2 x,-2 y, 0)$. Then, by $(2)$ the mean $\phi$-curvature of the ruled surface is

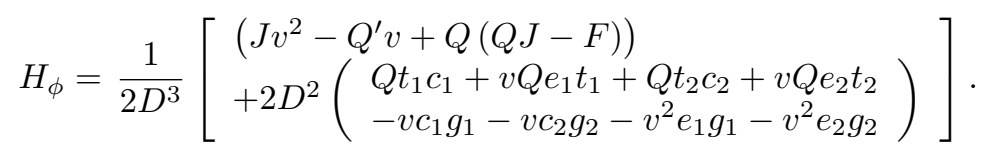

Corollary 1. Let the ruled surface $\varphi$ in space with density $e^{-x^{2}-y^{2}}$ be minimal surface. The offset ruled surface in space with density is minimal surface if and only if

$Q t_{1} c_{1}+v Q e_{1} t_{1}+Q t_{2} c_{2}+v Q e_{2} t_{2}-v c_{1} g_{1}-v c_{2} g_{2}-v^{2} e_{1} g_{1}-v^{2} e_{2} g_{2}=0$

where 


$$
\begin{aligned}
& v_{1}=\frac{-c_{1} g_{1}-c_{2} g_{2}+Q e_{1} t_{1}+Q e_{2} t_{2}+\sqrt{4\left(e_{1} g_{1}+e_{2} g_{2}\right)\left(c_{1} Q t_{1}+Q c_{2} t_{2}\right)+\left(c_{1} g_{1}+c_{2} g_{2}-Q\left(e_{1} t_{1}+e_{2} t_{2}\right)\right)^{2}}}{2\left(e_{1} g_{1}+e_{2} g_{2}\right)}, \\
& v_{2}=\frac{-c_{1} g_{1}-c_{2} g_{2}+Q e_{1} t_{1}+Q e_{2} t_{2}-\sqrt{4\left(e_{1} g_{1}+e_{2} g_{2}\right)\left(c_{1} Q t_{1}+Q c_{2} t_{2}\right)+\left(c_{1} g_{1}+c_{2} g_{2}-Q\left(e_{1} t_{1}+e_{2} t_{2}\right)\right)^{2}}}{2\left(e_{1} g_{1}+e_{2} g_{2}\right)} .
\end{aligned}
$$

With the help of the equation (2), the mean $\phi$-curvature of offset ruled surface $\varphi^{*}$ in space with density $e^{-x^{2}-y^{2}}$ is obtained

$$
\begin{aligned}
& H_{\phi}^{*}=\frac{1}{2 D^{* 3}}\left[\begin{array}{l}
\left(R^{2} J^{\prime}+F Q^{\prime}+F^{\prime} J R-F R J^{\prime}-R Q^{\prime}-F^{\prime} Q\right) \\
+\left(2 R J^{\prime}+F^{\prime} J-F J^{\prime}-Q^{\prime}\right) v+J^{\prime} v^{2}
\end{array}\right] \\
&-\frac{1}{2 D^{*}}\left((-2 x-2 y)\left[(-Q+J R+J v)\left(e_{1}, e_{2}, e_{3}\right)+(F-R-v)\left(g_{1}, g_{2}, g_{3}\right)\right]\right) .
\end{aligned}
$$

\section{Example}

Let a ruled surface be

$$
\varphi(u, v)=\left(\sin u+v \frac{4}{5} \cos u,-\frac{4}{5} \cos u+v \sin u, v \frac{3}{5} \cos u\right)
$$

in Euclidean 3- space $E^{3}$ with density. If $R=u$, parametric equation of evolute offset $\varphi(u, v)$ is

$\varphi^{*}(u, v)=\left(\sin u-\frac{4}{5} u \sin u-\frac{4}{5} v \sin u,-\frac{4 \cos u}{5}+u \cos u+v \cos u,-\frac{3}{5} v \sin u\right)$.

The Gaussian and mean curvatures of ruled surface $\varphi(u, v)$ and offset ruled surface $\varphi^{*}(u, v)$ are (see Figure $1-4$ )

$$
\begin{aligned}
K & =-\frac{900 \cos ^{2} u}{\left(9+50 v^{2}+9 \cos (2 u)\right)^{2}}, \\
K^{*} & =-\frac{900 \cos ^{2} u}{\left(41+50 u^{2}-80 v+50 v^{2}+20 u(-4+5 v)+9 \cos (2 u)\right)^{2}},
\end{aligned}
$$

and

$$
\begin{aligned}
H & =\frac{15 \sqrt{2}(4 \cos u-5 v \sin u)}{\left(9+50 v^{2}+9 \cos (2 u)\right)^{\frac{3}{2}}}, \\
H^{*} & =-\frac{15 \sqrt{2}(-4+5 u+5 v) \sin u}{\left(41+50 u^{2}-80 v+50 v^{2}+20 u(-4+5 v)+9 \cos (2 u)\right)^{\frac{3}{2}}},
\end{aligned}
$$

respectively. 
The Gaussian and mean $\phi$-curvatures of ruled surface $\varphi(u, v)$ and offset ruled surface $\varphi^{*}(u, v)$ in space with density $e^{-x^{2}-y^{2}}$ are

$$
\begin{aligned}
K_{\phi} & =-\frac{900 \cos ^{2} u}{\left(9+50 v^{2}+9 \cos (2 u)\right)^{2}}+4 \\
K_{\phi}^{*} & =-\frac{900 \cos ^{2} u}{\left(41+50 u^{2}-80 v+50 v^{2}+20 u(-4+5 v)+9 \cos (2 u)\right)^{2}}+4
\end{aligned}
$$

and

$$
\begin{aligned}
& H_{\phi}=-\frac{3\left(-20\left(80+27 v+100 v^{3}\right) \cos u-180 v \cos (3 u)+2\left(27+1000 v+100 v^{2}+4\left(9+25 v^{2}\right) \cos (2 u)+9 \cos (4 u)\right) \sin u\right)}{40 \sqrt{2}\left(9+50 v^{2}+9 \cos (2 u)\right)^{\frac{3}{2}}}, \\
& H_{\phi}^{*}=\frac{3\left(\cos u(-1-10 u-10 v+\cos (2 u))\left(41+50 u^{2}-80 v+50 v^{2}+20 u(-4+5 v)+9 \cos (2 u)\right)-100(-4+5 u+5 v) \sin u\right)}{10 \sqrt{2}\left(41+50 u^{2}-80 v+50 v^{2}+20 u(-4+5 v)+9 \cos (2 u)\right)^{\frac{3}{2}}},
\end{aligned}
$$

respectively.

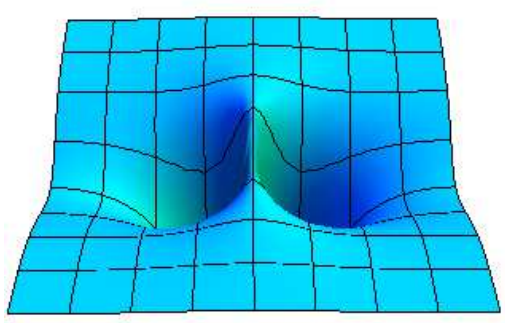

Figure 1: Gaussian curvature $K$ of ruled surface $\varphi(u, v) 0 \leq \mathrm{u} \leq \pi,-1 \leq \mathrm{v} \leq$ 1.

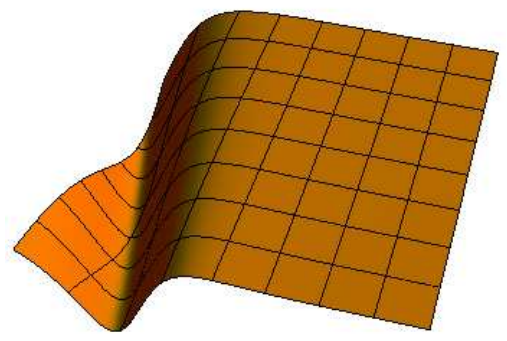

Figure 2: Gaussian curvature $K^{*}$ of ruled surface $\varphi^{*}(u, v), 0 \leq \mathrm{u} \leq \pi, 0 \leq \mathrm{v}$ $\leq 1$. 


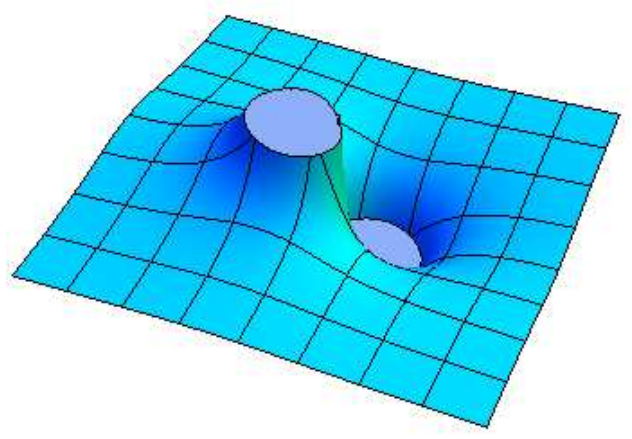

Figure 3: Mean curvature $H$ of ruled surface $\varphi(u, v), 0 \leq \mathrm{u} \leq \pi,-1 \leq \mathrm{v} \leq 1$.

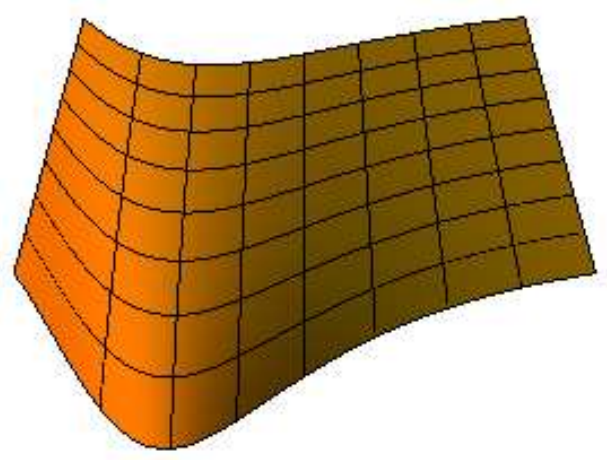

Figure 4: Mean curvature $H^{*}$ of ruled surface $\varphi^{*}(u, v), 0 \leq \mathrm{u} \leq \pi, 1 \leq \mathrm{v} \leq 2$ 


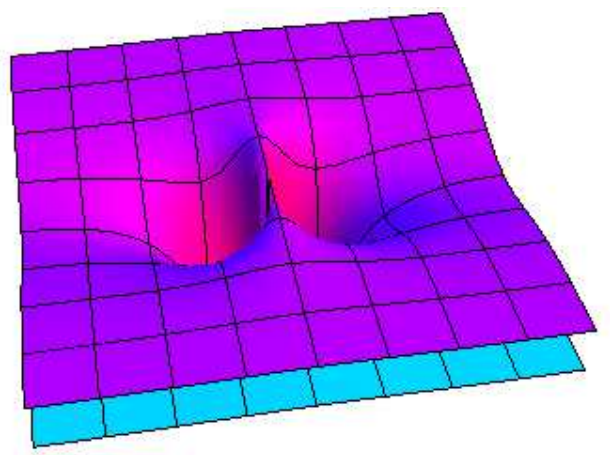

Figure 5: The relationship between Gaussian curvature $K$ of ruled surface $\varphi(u, v)$ and Gaussian $\phi$-curvature $K_{\phi}$ of ruled surface $\varphi(u, v)$ in space with density $e^{-x^{2}-y^{2}}, \quad 0 \leq \mathrm{u} \leq \pi, \quad-1 \leq \mathrm{v} \leq 1$.

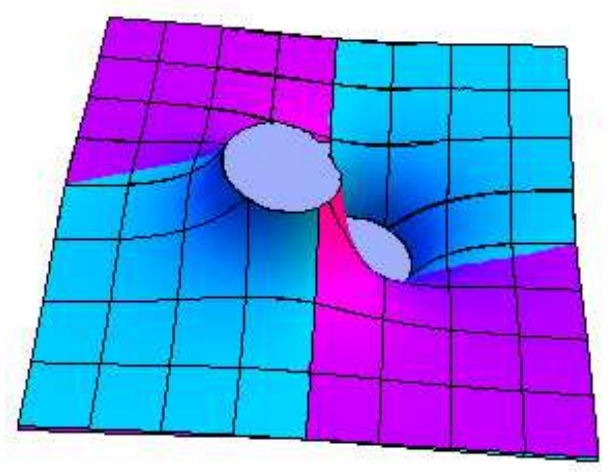

Figure 6: The relationship between mean curvature $H$ of ruled surface $\varphi(u, v)$ and mean $\phi$-curvature $H_{\phi}$ of ruled surface $\varphi(u, v)$ in space with density $e^{-x^{2}-y^{2}}, \quad 0 \leq \mathrm{u} \leq \pi, \quad-1 \leq \mathrm{v} \leq 1$. 


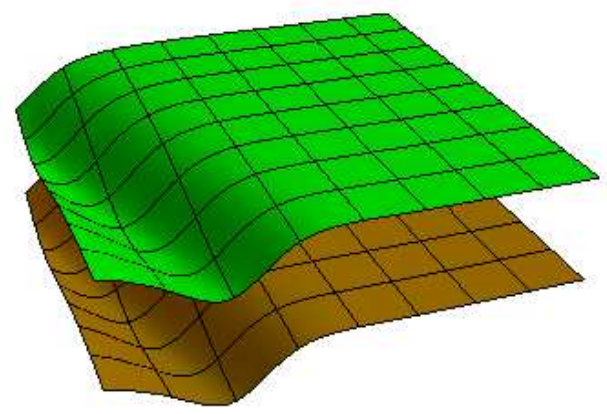

Figure 7: The relationship between Gaussian curvature $K^{*}$ and Gaussian $\phi$ curvature $K_{\phi}^{*}$ of offset ruled surface $\varphi^{*}(u, v)$ in space with density $e^{-x^{2}-y^{2}}, 0$ $\leq \mathrm{u} \leq \pi, \quad 0 \leq \mathrm{v} \leq 1$

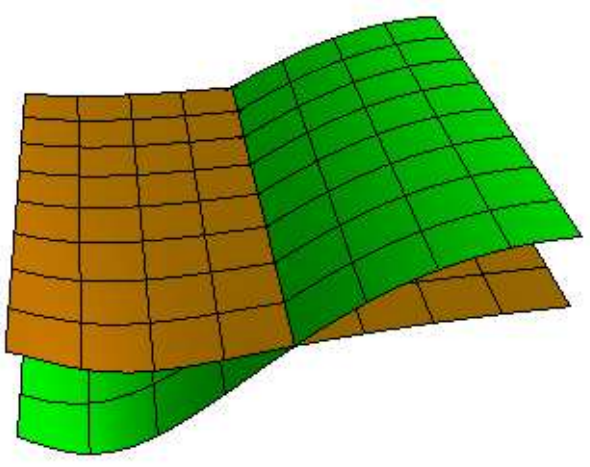

Figure 8: The relationship between mean curvature $H^{*}$ and mean $\phi$-curvature $H_{\phi}^{*}$ of offset ruled surface $\varphi^{*}(u, v)$ in space with density $e^{-x^{2}-y^{2}}, 0 \leq \mathrm{u} \leq \pi$, $1 \leq \mathrm{v} \leq 2$. 


\section{References}

[1] F. Morgan, Manifolds with Density. Notices of AMS. 52(8) (2005), 853858 .

[2] F. Morgan, Myers' Theorem with Density, Kodai Mathematical Journal. 29(3) (2006), 455-461.

[3] F. Morgan, Manifolds with Density and Perelman's Proof of the Poincaré Conjecture, The American Mathematical Monthly. 116(2) (2009), 134-142 .

[4] C. Rosales, A. Canete, On the isoperimetric problem in Euclidean Space with density, Calculus of Variations. 3 (2008), 27-46.

[5] I. Corwin, N. Hoffman, S. Hurder, V. Sesum, Y. Xu, Differantial Geometry of Manifolds with Density. Rose-Hulman Undergraduate Mathematics Journal. 7(1) (2006), https://scholar.rosehulman.edu/rhumj/vol7/iss1/2.

[6] L. Belarbi, M. Belkhelfa, Surfaces in $R^{3}$ with Density. i-managers Journal on Mathematics. 1(1) (2012), 34-48.

[7] R. Lopez, Minimal Surfaces in Euclidean Space with a Log-linear Density, https://arxiv.org/abs/1410.2517 (April 01, 2019).

[8] D.S. KIm, D.W. Yoon, Constructions of Helicoidal Surfaces in Euclidean Space with Density, Symmetry. 9(173) (2017), doi:10.3390/sym9090173.

[9] D.T. Hieu, N.M. Hoang, Ruled Minimal Surfaces in $R^{3}$ with Density $e^{z}$, Pacific J. Math. 243(2) (2009), 277-285.

[10] Ö.G. Yildiz, S. Hizal, M. AkyıĞIT, Type $I^{+}$Helicoidal Surfaces with Prescribed Weighted Mean or Gaussian Curvature in Minkowski Space with Density, An. Sit. Univ. Ovidius Constanta. 26(3) (2018), 9-108.

[11] Ö.G. YILDIZ, Constructions of Helicoidal Surfaces in a 3dimensional Complete Manifold with Density, Mathematics. 7(1) (2019) https://doi.org/10.3390/math7010027.

[12] M. Altin, A. Kazan and H.B. Karada $\breve{G}$, Rotational Surfaces Generated by Planar Curves in $E^{3}$ with Density, Int. J. Anal. Appl. 17(3) (2018), 311-328. 
[13] D. W. Yoon, Z. Kucukarslan Yuzbasi, Weighted Minimal Affine Translation Surfaces in Euclidean Space with Density, Int. J. Geom. Methods Mod. Phys. 15(11) (2018), 1850196.

[14] A. Kazan, H.B. Karada $\breve{G}$, Weighted Minimal and Weighted Flat Surfaces of Revolution in Galilean 3-space with Density, Int. J. Anal. Appl. 16(3) (2018), 414-426.

[15] D. W. Yoon, On the Offsets of Ruled Surfaces in Euclidean Space, Int. J. Geom. Methods Mod. Phys. 108(4) (2016), 985-997.

[16] F. Guler, E. Bayram, E. Kasap, Offset Surface Pencil with a Common Asymptotic Curve, Int. J. Geom. Methods Mod. Phys. 15(11) (2018), 1850195.

Neslihan ULUCAN,

Department of Mathematics,

Sakarya University,

54187 Sakarya, Turkey.

Email: neslihancabaci@gmail.com

Mahmut AKYIGIT,

Department of Mathematics,

Sakarya University,

54187 Sakarya, Turkey.

Email: makyigit@sakarya.edu.tr 